\title{
Minefield Mapping Using Cooperative Multirobot Systems
}

\author{
Alaa Khamis ${ }^{1,2}$ and Asser ElGindy ${ }^{1}$ \\ ${ }^{1}$ Robotics and Autonomous Systems (RAS) Research Group, German University in Cairo, New Cairo, Cairo 11835, Egypt \\ ${ }^{2}$ Engineering Science Department, Suez Canal University, Suez, 43721, Egypt \\ Correspondence should be addressed to Alaa Khamis, akhamis@pami.uwaterloo.ca and Asser ElGindy, asser.elgindy@gmail.com \\ Received 30 June 2012; Revised 11 September 2012; Accepted 17 October 2012 \\ Academic Editor: Huosheng $\mathrm{Hu}$ \\ Copyright (C) 2012 A. Khamis and A. ElGindy. This is an open access article distributed under the Creative Commons Attribution \\ License, which permits unrestricted use, distribution, and reproduction in any medium, provided the original work is properly \\ cited. \\ This paper presents a team-theoretic approach to cooperative multirobot systems. The individual actions of the robots are \\ controlled by the Belief-Desire-Intention model to endow the robots with the know-how needed to execute these actions \\ deliberately. The cooperative behaviors between the heterogeneous robots are governed by the Team-Log theory to endow all \\ the robots in the team with the know-how-to-cooperate and determine the team members' commitments to each other despite \\ their different types, properties, and goals. The proposed approach is tested for validity with the real life problem of minefield \\ mapping. Different minefield sweeping strategies are studied to control the mobility of the mobile sweepers within the minefield \\ in order to maximize the area coverage and improve picture compilation capability of the multirobot system.
}

\section{Introduction}

Developing a robust and cooperative team of robots capable of solving complex tasks is an interesting area of research that attracts many researchers nowadays. Achieving robust and productive cooperation between various system components is inspired by different domains such as biology, artificial life, psychology, and cognitive science in order to build artificially cooperative intelligent systems. Cooperation is defined in [1] as a purposive positive interference of agents to further the achievement of a common goal or goals compatible with their own. To achieve this effective cooperation in multirobot systems (MRS), the robots must have know-how for solving simple problems in an autonomous way and a know-howto-cooperate by which agents can share common interests and interact with each other to solve complex problems cooperatively.

In recent years, scientific community has seen a great number of research works dedicated to cooperative multirobot systems and their applications in different areas such as search and rescue $[2,3]$, distributed surveillance [4], communication relaying [5], agriculture [6], sorting
[7], emergency services [8], and landmine detection [9]. Minefield reconnaissance and mapping is one of the most promising applications of cooperative multirobot systems.

In the context of humanitarian demining, cooperative multirobot systems can be beneficial for deminers, civilians, and government. The design of an accurate sensor may reduce the amount of time needed to determine whether a landmine exists, but does not increase the safety of the deminer. Since the safety issues during the eradication process are of great concern, the use and integration of cheap and simple mobile sweepers in cooperative multirobot system can provide a promising solution. Cooperative multirobot systems can replace the human deminers providing a safer, more accurate way for mine mapping. Given the fact that landmines are indiscriminate killers that target civilians long after a conflict has ended, the accurate maps created by cooperative multirobot systems can be used to remove or deactivate the detected mines promoting a safe access for the civilian to the cleared lands. Moreover, major effect of mines is to deny access to rich lands, and their resources. Besides this, the medical, social, economic, and environmental consequences are immense. Cooperative multirobot systems 
can play a crucial role in solving the problem of landmines promoting safe access to lands to develop new projects that contribute to a country's development.

In this paper, a team-theoretic approach to a cooperative multirobot system is proposed for minefield mapping. The cooperative behaviors between heterogenous robots are designed to assist in humanitarian demining mission. For this mission, the multirobot system encompasses a number of sensing agents (mine sweepers to detect the mines) and acting agents (deminers to remove or deactivate the mines). Belief-Desire-Intention model is used to control the actions of the robots and the relation between the team members is governed by the Team-Log formalism that determines the team members' commitments to each other. The multirobot system outputs a clustered mine map by using genetic algorithm. Then this clustered map is passed to a number of deminers to successfully remove or deactivate the landmines.

The remainder of this paper is organized as follows: Section 2 introduces cooperative multirobot systems in the context of humanitarian demining highlighting their features and discussing the cooperation aspect of these systems. Section 3 describes the know-how and the knowhow-to-cooperate in multirobot systems. The proposed teamtheoretic approach is presented in Section 4 followed by describing its application for minefield mapping in Section 5. Finally conclusion and future work are summarized in Section 7.

\section{Cooperative Multirobot Systems in Humanitarian Demining Context}

The problem of landmines is considered a severe problem in many countries in the world which faced many wars during the past century. Recent studies show that Egypt alone has over 20-23 million landmines distributed on $22 \%$ of its total surface area thus making it one of the most contaminated countries in the whole world [10]. These landmines make the contaminated area almost unusable and nearly impossible to benefit from it until the land mines are cleared. Moreover as the years pass clearing the landmines becomes more and more difficult due to the accumulation of obstacles because of the changing weather conditions. In addition the process of clearing landmines is usually dangerous for humans and consumes much time and resources [11]. In this case, a cooperative multirobot system can serve as a solution to this problem.

Multirobot system (MRS) is a group of robots that are designed aiming to perform some collective behavior. The MRS is gaining great interest because of the following reasons [12].

(i) Resolving task complexity: some tasks may be quite complex for a single robot to do or even it might be impossible such as box pushing or crossing over a gap. Other tasks are inherently distributed such as distributed monitoring, reconnaissance, and surveillance.

(ii) Increasing system performance: multiple robots can solve problems faster using parallelism. This can lead to maximizing object, area, or radio spatial/temporal coverage and minimizing task completion time.

(iii) Reliability: increasing the system reliability because of having only one robot may work as a bottleneck for the whole system especially in critical times. However when using multiple robots to do a task and one fails, other robots could still do the job. Therefore, the introduction of multiple robots increases robustness through redundancy.

(iv) Simplicity in design: building several resourcebounded robots is much easier and sometimes more economical than building a single powerful robot.

The advantages of using MRS in humanitarian demining can be summarized as follows [13].

(i) Minefields are dangerous to humans; a robotic solution allows human operators to be physically removed from the hazardous area.

(ii) The use of multiple, inexpensive robotized search elements minimizes damage due to unexpected exploding mines and allows the rest of the mission to be carried on by the remaining elements.

(iii) Many kinds of mines must be dealt with; the use of many robots allows mines to be pursued in parallel, rather than one at a time.

(iv) A number of sensors have been developed whose information can be related to the presence or absence of mines. None of these sensors on their own is however sufficient and hence some combination of these sensors must be used.

In order to demine effectively, the robots should exhibit the following skills.

(i) Follow the defined search pattern.

(ii) Negotiate difficult terrain.

(iii) Record and report position during the whole mission.

(iv) Work fast enough to be cost-effective.

(v) Avoid interference with each other.

(vi) Cover the terrain effectively.

(vii) Share workload.

(viii) Help each other by providing complementary information via different sensors.

(ix) To be capable of dynamic redistribution of the workload in case of robot failures.

Strategies for multirobot navigation require robots capable at least of some basic behaviors such as avoiding obstacles, finding the mines, following a specific path, and maintaining a formation. In [13], three basic behaviors (avoiding obstacles, achieving a goal, for maintaining the position in a specific formation, and maintaining the robot direction) to create more complex behaviors are used for mine detection such as random movement, relay clustering, 
flocking, swarming, formation maintenance, and comb movement. Another social foraging theory-based approach is proposed in [14]. In this system the robots represent the foragers and the mines will be the preys. The foragers (the robots) will have to scan cooperatively all the minefield in order to localize all the preys (the mines).

Agents are well suited for use in applications that involve distributed computation or cooperation between components which is the case of multirobot systems as the system tries to decompose a global task into subcomponents and establish cooperation mechanisms by which these subcomponents can work together effectively, synergically and harmoniously. Agents are expected to perceive the surrounding environment and execute certain actions on it until a final state is achieved. These autonomous agents, while operating on local knowledge and possessing only limited abilities, are nonetheless capable of enacting the desired global behaviors [15]. In MRS, cooperation is purposive positive interference of agents to further the achievement of a common goal or goals compatible with their own [1]. Their forms of cooperation can be manifested in these systems, namely, augmentative, integrative, and debative $[10,16]$.

(i) Augmentative Cooperation. Agents have a similar know-how, but the agents must be multiplied to perform a task that cannot be performed by only one agent. This task is then shared into similar subtasks. In the context of minefield mapping, cooperation between mobile sweepers with similar know-how to create a mine map is considered as augmentative cooperation.

(ii) Integrative Cooperation. Agents have different and complementary know-how and it is necessary to integrate their contribution for reaching a predefined goal. Moreover the agent types and physical properties can be different from each other, thus every type of agent is responsible for a task that may be different than other agents in the same team. In humanitarian deminiingg, cooperation between sensing agents (mobile sweepers) and acting agents (deminers) is an example for integrative cooperation.

(iii) Debative Cooperation. When agents have a similar know-how and are faced with a unique task again, this time they are forced to compare their results for obtaining the best solution from all the solutions of each agent. The agents must be aware of the fitness function on which they are going to select the best solution with respect to their understanding of this function. In minefield mapping, sensing agents can cooperate debatably in order to select the most appropriate agent to replace a failed agent.

This multiagent system paradigm introduces a number of new design/development issues when compared with more traditional approaches to systems development. However, the following challenging aspects must be addressed.

(i) How to make agents able to decompose their goals and tasks, to allocate subgoals and subtasks to other agents, and to synthesize partial results and solutions [17].

(ii) How to enable agents to communicate. What communication languages and protocols to use [17].

(iii) How to effectively balance local computation and communication [18].

(iv) How to enable agents to represent and reason about the actions, plans, and knowledge of other agents in order to appropriately interact with them [17].

(v) How to enable agents to represent and reason about the state of their interaction processes. How to enable them to find out whether they have achieved progress in their coordination efforts, and how to enable them to improve the state of their coordination and to act coherently [17].

(vi) How to enable agents to recognize and reconcile disparate viewpoints and conflicts. How to synthesize views and results [17].

(vii) How to enable agents to negotiate and contract. What negotiation and contract protocols they use [19].

(viii) How to formally describe multiagent systems and the interactions among agents. How to make sure that they are correctly specified [19].

(ix) How to realize "intelligent" processes such as problem solving, planning decision making, and learning in multiagent contexts. How to enable agents to collectively carry out such processes in a coherent way [19].

Hence, new analysis and design methodologies and new tools are needed to create an effective multirobot systems using multiagent paradigm. In the context of humanitarian demining, the cooperative multirobot system will encompass a number of spatially distributed agents that, when properly managed, can collectively and continuously scan a minefield and build a mine map. These agents are endowed with knowhow capability for solving problems in an autonomous way and a know-how-to-cooperate capability by which the agents can share common interests and interact with each other. Each subarea will be scanned by a mobile sweeper. The following section describes in details the know-how and the know-how-to-cooperate of the agents.

\section{Know-How and Know-How-to-Cooperate}

3.1. Know-How. In the context of humanitarian demining, the robot or agent has to be aware of how it is going to achieve its goals and finish the task. To complete the goal the task has to be divided into smaller actions. This implies that the agent has to know what are these small actions? When are they going to be executed? How they are going to be executed? The answer of these questions is the know-how by which the agent will have to solve problems autonomously. The knowhow gives a model of the individual activity of an agent. An agent cannot be guaranteed to succeed with its intentions if it lacks the know-how to achieve them [20]. 
Artificial agents build their know-how by performing four main activities, namely: information gathering/elaboration, diagnosis, intervention decision, and action [21]. BeliefDesire-Intention (BDI) model can be used to implement the agent's know-how. A BDI agent has beliefs about itself, other agents, and its environment desires about future states (i.e., goals), and intentions about its own future actions (i.e., plans). This model of human practical reasoning was first developed by Bratman [22] as a method to explain the future-directed intention. When using a software model for developing an intelligent agent, it implements the agent's beliefs, desires, and intentions and actually uses these concepts to solve a particular problem in agent programming. Essentially, it works on selecting the appropriate plan to be applied based on the current believes and desires independent of the currently active plans. Hence, BDI agents are capable of balancing the time spent on selecting which plan to execute and actually executing those plans.

Georgeff et al. have adopted this model and transformed it into a formal theory and an execution model for software agents, based on the notion of beliefs, goals, and plans [23]. The concepts of the BDI model and how it works will be presented in this section. BDI model abstracts the following concepts.

(i) Beliefs. The agent beliefs represent the current information the agent possesses, in other words its understanding of the surrounding environment (including itself and other agents). Beliefs capture informational attitudes [24]. They are propositions about the state of the world they consider based on their perceptions [25]. Beliefs can also include inference rules, which allow forward chaining to reach new beliefs based on the old belief the agent had. The term belief is used instead of knowledge to imply that what an agent currently believes might not be fact or constant but may change afterwards due to new updates.

(ii) Desires. Desires represent the motivational attitudes [24]. They are propositions about the world that they would like to be true [25]. They represent objectives the agent would like to accomplish or states to reach. Examples of desires might be, minimum scanning time, maximum coverage, or minimum number of sensors.

(iii) Goals. A goal is a desire that has been selected to be reached by the agent. The term goals adds more restriction to the meaning, such that the set of active desires must be consistent. For example, one should not have the two goals of maximizing coverage and minimizing number of sensors at the same time; however, both could be desirable from the agent at any time.

(iv) Intentions. Intentions are the deliberative attitudes of agents [24]. Intentions are desires to which the agent has to some extent committed it self. In implemented systems, this means the agent has begun executing a plan [25]. (v) Plans. Plans are sequences of small actions that the agent has to perform to achieve one or more of its intentions. They have all the details that the agent needs to know to be able to successfully achieve his intention. Plans may include other plans: my plan to go detect landmines may include a plan to scan certain area of interest. This means that in Bratman's model, plans are initially only partially conceived, with details being filled in as they progress.

(vi) Events. These are triggers for the agents to do something. An event may update beliefs, trigger plans or modify goals. Events can be either generated externally and received by sensors or can be generated internally to trigger decoupled updates or plans of activity.

3.2. Know-How-to-Cooperate. The know-how-to-cooperate allows agents to manage interference between their goals, resources, and so forth and allows agents to perform their own activities taking into account the activities of the other agents [21]. Each agent relies on its knowledge of other agents and the domain to achieve a high degree of efficiency in reaching both local and global goals. This interaction is characterized by mutual interest and only a partial knowledge of the reasoning steps of the other agents and of the environment. The goals might or might not be known to the agents explicitly, depending on whether or not they are goal directed. Goal-directed agents may also change their goals to suit the needs of other agents in order to ensure cohesion and coordination. Different models have been proposed to achieve the know-how-to-cooperative such as Joint Responsibility model [26], Joint Intentions [27], Satisfaction-Altruism model [28], Soar model [29], and TeamLog model [30]. In this section the TeamLog theory [30] is used to achieve the know-how-to-cooperate in a team of heterogeneous agents.

3.2.1. Beliefs in Groups. The language used is following the standard $K D 45_{n}$-system for $n$ agents is explained in $[31,32]$.

(i) General Belief. The formula $E \_B E L G(\varphi)$, called "general belief" means every agent in group $G$ believes $\varphi$. That is, $\mathrm{M}, \mathrm{s} \vDash$ ? $E \_B E L_{G}(\varphi)$ if and only if for all $i \in G, M, s \vDash ? B E L_{G}(i, \varphi)$ which corresponds to axiom $1:$

$$
\begin{aligned}
& \text { Axiom } 1(\text { GeneralBelief }) \quad: \quad E_{B E L G}(\varphi) \leftrightarrow \\
& \wedge_{i \in G} B E L(i, \varphi) .
\end{aligned}
$$

(ii) Common Belief. The traditional way of upgrading single agent concept to multi-agent systems is through the use of common belief $C B E L_{G}(\varphi)$ between the group members. $C \_B E L_{G}(\varphi)$ is considered to be true if everyone in $G$ believes $\varphi$, and everyone in $G$ believes that everyone else also believes $\varphi$. This is a rather stronger operator than general belief, even though $\varphi$ 
does not need to be true. The corresponding axiom is shown in Axiom 2:

$$
\begin{aligned}
& \text { Axiom } 2(\text { CommonBelief }) \quad: \quad C \_B E L_{G}(\varphi) \leftrightarrow \\
& E_{B E L} L_{G}\left(\varphi \wedge C \_B E L_{G}(\varphi)\right) .
\end{aligned}
$$

3.2.2. Collective Intentions. Intentions form a rather special consistent subset of goals, that is, it is viewed as a chosen goal; providing inspiration for a more concrete social commitment in the individual case and a plan-based collective commitment in the group case $[22,33]$. There are several discussions on intention from different viewpoints; a common agreement is summarized as

(i) intentions drive means-end-reasoning (how to achieve a goal),

(ii) intentions constrain future deliberation,

(iii) intentions persist long enough, according to a reconsideration strategy,

(iv) intentions influence beliefs upon which future practical reasoning is based.

While the mechanism behind group intentionality might still be mysterious from the psychological viewpoint, the logical modeling is worthy to allow a team of agents to cooperate smoothly $[34,35]$.

(i) Language for goals and intentions: if $\varphi$ is a formula, $i \in A$ and $G \subseteq A$, then the following are formulas: $\operatorname{GOAL}(\mathrm{i}, \varphi), \operatorname{INT}(\mathrm{i}, \varphi), E_{\_} I N T_{G}(\varphi), M \_I N T_{G}(\varphi)$, $C \operatorname{INT}_{G}(\varphi)$.

(ii) General, mutual and collective intentions: What motivates a group of agents to combine their efforts to achieve a common goal $\varphi$ ?

The first level is called general intention, in which everyone in the group $G$ individually intends $\varphi$, and is expressed as

$$
E_{I N T} T_{G}(\varphi) \longleftrightarrow \bigwedge_{i \in G} I N T(i, \varphi) .
$$

Mutual intention means that recursively everyone intends that everyone intends $\varphi$, and is expressed as

$$
M \_I N T_{G}(\varphi) \longleftrightarrow E I N T_{G}\left(\varphi \wedge M \_I N T_{(\varphi)}\right) .
$$

The collective intention is a much stronger concept, which means that everyone has the mutual intention and everyone believes in the existence of the mutual intention, and is expressed as:

$$
C_{I} I N T_{G}(\varphi) \longleftrightarrow M \_I N T_{G}(\varphi) \wedge C \_B E L_{G}\left(M \_I N T_{G}(\varphi)\right) .
$$

3.2.3. Collective Commitments. Suppose we have a team with collective intention to achieve a goal $\varphi$. It is not sufficient for the team to start its cooperative action towards this goal. We need a bridge that connects the intentions with the detailed actions. Collective commitment in a group of agents is aimed to trigger a specific team action, that is, a coordinated execution of agents' individual actions according to the selected social plan.

The meaning of teamwork covers different gradations of being a team, ranging from loosely coupled team such as a research group, to tightly organized team. The idea of tuning the nature of commitment is intuitively appealing because both the circumstances in which the agents are acting and the structure of their organization are constantly dynamically changing.

Group commitments are comprised of the following three aspects.

(1) Group: usually, a cooperative team of agents has to be formed on the basis of a collective intention.

(2) Plan: a social plan that details how to realize the group's goal needs to be created.

(3) Distribution of responsibilities: a set of pair-wise social commitments towards the actions from the social plan reflects the agents' responsibilities during team actions.

(1) Social plan: Collective commitments are based on plans: they are defined with respect to a given social plan. Individual actions, are combined into group actions by social plan expressions. The social plan leads to the successful realization of achieving a certain goal $\varphi$.

(2) Social commitment: Collective commitments are realized through pair-wise commitments towards the actions. For a simple case, cooperation between two agents involves a certain asymmetric role division: the first agent $i$ wants an action to be performed while a second agent $j$ decides that it can perform the action needed. When $i$ is willing to have $j$ as a helper and to oversee the achievement of the goal, they recognize their potential for cooperation. This recognition is reflected in a promise from $j$ to $i$. Thus, a social commitment is the bilateral motivational attitude that corresponds to such a promise.

\section{Team-Theoretic Approach}

A team-theoretic approach is proposed to develop cooperative behaviors in multirobot systems. Based on this approach, each robot will be equipped with BDI-based know-how capability for solving problems in an autonomous way and a TeamLog-based know-how-to-cooperate capability by which the robots can share common interests and interact with each other.

The proposed system is based on a multilevel architecture where agents are conceptually arranged in a tree-like structure. In this structure, agents higher in the tree have a more global view than those below them. In its strictest interpretation, interactions do not take place across the tree, but only between connected entities [36]. The data produced by lower-level agents in a hierarchy typically travels upwards to provide a broader view, while control flows downward as 
the higher level agents provide direction to those below [17] as illustrated in Figure 1.

The architecture is divided into three levels: Sensing agents and acting agents, micromanagers and macromanager. Each agent has his own set of beliefs, desires, and intentions. Let $\mathbf{w}$ be the total number of sensing agents, $\mathbf{m}$ be the total number of acting agents, $\mathbf{n}$ number of Micromanagers, and $\mathbf{k}$ number of sensing agents assigned to micromanager.

Following this multilevel architecture, the proposed system encompasses the following spatially distributed agents.

(i) Sensing Agents. The sensing agents are deployed across the environment and are responsible of detecting and recording certain objects/events in this environment. They are equipped with the know-how needed to complete its main task by performing small actions. Examples of these basic actions are, move-to, rotate, avoid obstacles and report event/object.

(ii) Acting Agents. The acting agents are specialized agents responsible of manipulating the environment after the sensing agents have finished the scanning task and they are equipped as well with the know-how to physically manipulate the environment to reach the desired state of environment.

(iii) Micromanagers. Micromanagers are the direct managers of the sensing agents; they are equipped with the know-how to report the local map to macromanager as well as the know-how-to-cooperate to be able to manage their sensing agents via delegation and also communicate with the macromanager.

(iv) Macromanager. Macromanagers are the managers of the micromanagers. They are also equipped with the know-how to combine the local maps into a global map for the area of interest and the know-howto-cooperate to coordinate between the different micromanagers and the acting agents via delegation. The user of the proposed system will interface with this macromanager.

4.1. BDI-Based Know-How. The BDI model described in the previous section has been used to implement the agent know-how that allows the sensing agents to solve problems in an autonomous manner.

(i) Beliefs and Knowledge. The beliefs of the sensing agent describe its knowledge of its position, area of interest (AOI), individual map as well as its corresponding micromanager. The beliefs of the micromanager include its position, sensing agents, region of interest (ROI), local map and the macromanager. The beliefs of the macromanager include the entire environment, all micromanagers, all sweeping agents, all acting agents, and the global map before and after clustering.

(ii) Desires and Goals. In the proposed system the main global desire is to scan and to create a map for the entire environment by dividing it into smaller regions of interest and assigning each ROI to its respective micromanager. Afterwards the macromanager creates another desire of clustering the global map. The desires of the micromanagers are to divide its ROI into smaller areas of interest (AOI) and to report the local map to the macromanager. The desire of the sensing agents is to scan the AOI and report to micromanager if a certain object is found and of course avoid any obstacles in the AOI.

(iii) Intentions and Plans. The global plan is how exactly the macromanager will accomplish the global desire of scanning the environment disregarding the small details about the sensing agents. The micromanagers have their projection of the global plan called the social plan which is how to scan their ROI, subsequently the sensing agents have their own projection of the social plan to scan their AOI called the individual plan.

Algorithm 1 describes the BDI for the macromanager showing the main procedures and the order of the desires: the inputs to the macromanager are the micromanagers as agents, their number $\mathbf{n}$ and the number of acting agents $\mathbf{i}$.

Algorithm 2 describes the BDI of the micromanagers: the inputs to the micromanager are its global ID defined by the macromanager, its assigned ROI to be scanned, the sensing agents, and their number $\mathbf{k}$.

Algorithm 3 describes the BDI model for each sensing agent with brief information about its plan: the inputs are the current position of the agent on the grid, the unique ID, the assigned AOI, the micromanager responsible for this agent, and finally the readings from its sensors.

\subsection{TeamLog-Based Know-How-to-Cooperate}

(i) Global Plan: in order to control the amount of interactions and decrease the time needed to establish beliefs, the accepted team model is hierarchical. The macromanager views a team as a single sensing agent, even though the micromanagers use many sensing agents to perform their work.

(ii) Social Plan: the social plan for which the macromanager and micromanagers are responsible is designed with respect to certain environment. It is a whileloop, in which observation is interleaved with treatment of current state. The social plan of the micromanagers is how to scan the ROI given the current number of sensing agents per each micromanager.

(iii) Collective Intention: the group of micromanagers have a collective intention of dividing and reporting the detected objects/events. This expression is denoted by $C I N T_{G}(\varphi)$, where $G$ is the group of agents and $\varphi$ is the desired intention. The group of sensing agents also have a collective intention of scanning and reporting the detected objects/events.

(iv) Collective Commitment: as classified by [24] the type of collective commitment used is team commitment because each member of the subteam is aware of 


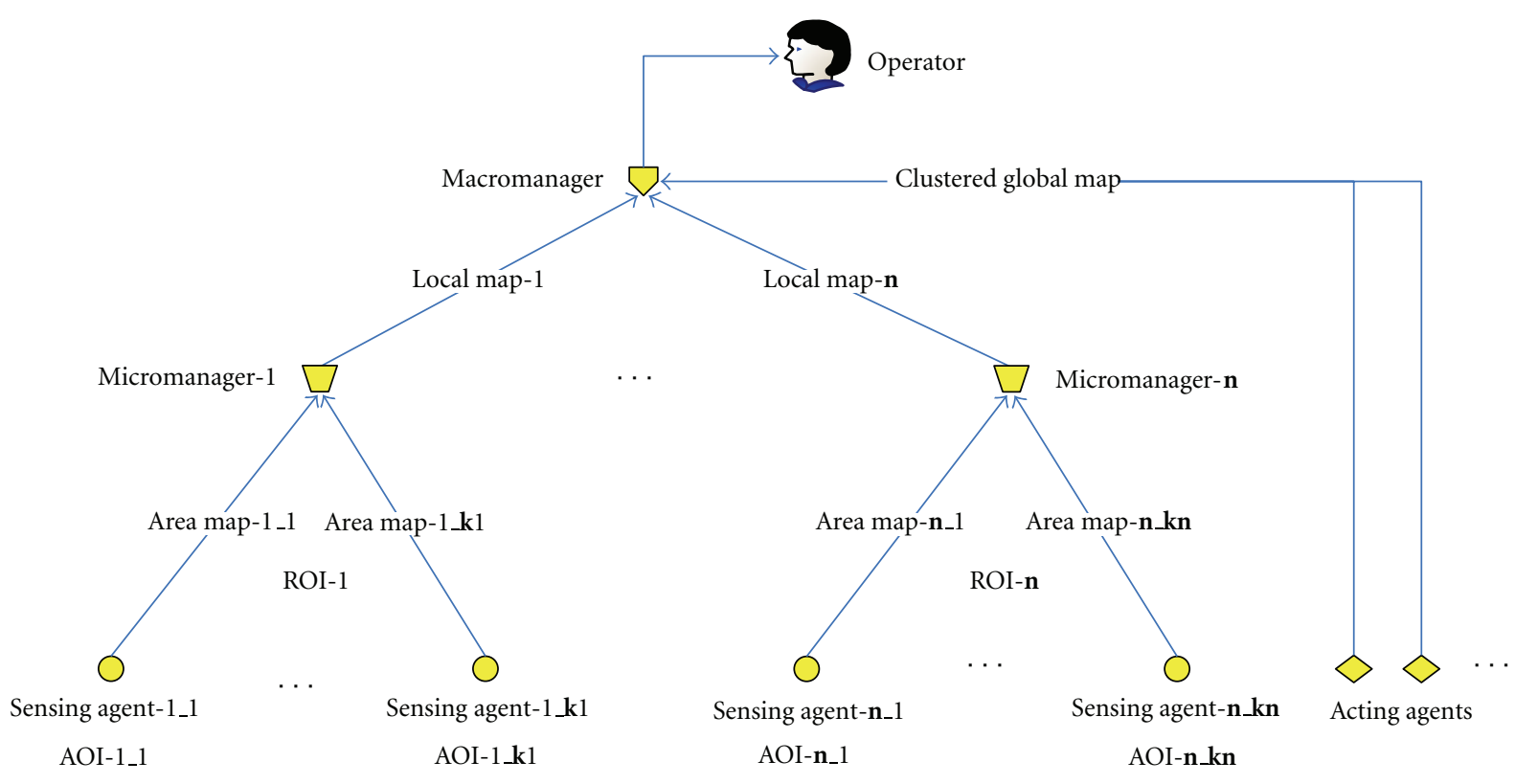

FIgURe 1: System levels.

(1) Beliefs micromanagers, $\mathbf{n}, \mathbf{i}$

(2) Perform divide Environment

(3) micromanager_ROI $\leftarrow$ Environment $\div \mathbf{n}$

(4) While Environment not scanned do

(5) Perform scan Environment

(6) Update Beliefs

(7) end while

(8) Perform generate global object/event map

(9) Perform cluster global map, i

Algorithm 1: Macromanager BDI model.

the goal but not aware of the other teams goals or commitments.

(v) Agent-to-Agent Commitments: the sensing agents are committed to their micromanager to complete the scanning and report an individual object map, moreover, the micromanager agents are committed to the macromanager to report a local object/event map for each micromanager. Finally the macromanager compiles the global object/event map based on the many local maps received from the different micromanagers then the acting agents are committed to manipulate the object/event as required by the macromanager.

4.3. Mobility Models. Two types of mobility models were implemented. Both types depend on the divide-and-conquer algorithm where a certain sensing agent is assigned only to one small AOI and neglects all other regions. Each sensing agent knows what are the boundaries of its AOI, but the difference is in how it is going to be scanned [37-39]. (i) Fully Coordinated Motion. As shown in Figure 2, the sensing agent finishes the scanning by moving in the AOI line by line horizontally. It starts from the top left corner of its $\mathrm{AOI}$ and starts moving right till it reaches the right boundary, then it moves one step downward, then it starts moving left till it reaches the left boundary and so on until the entire AOI is scanned. If an object/event is detected, the sensing agent has to record its location then follow a certain path to avoid it. Figure 2 shows an example of two sweepers scanning their AOI by following the line-by-line scanning mode, the green area is the sweeper's AOI, the first blue dot is the current position of the sweeper, and the blue dots are the recently visited positions. There are some disadvantages that prevent the fully coordinated motion strategy being widely practiced [40].

(1) The region of AOI may not be able to, or very difficult to, divide equally.

(2) Executing the perfect scanning plan needs employing a very accurate navigation system. Navigational inaccuracies due to sensor and actuator's tolerance lead 
(1) Beliefs ID, ROI, sensing agents, $\mathbf{k}$

(2) Initialize local map

(3) while ROI not scanned do

(4) Perform divide ROI

(5) agent_AOI $\leftarrow R O I \div \mathbf{k}$

(6) if agent found object/event then

(7) Update local object/event map

(8) end if

(9) Update Beliefs

(10) end while

Algorithm 2: Micromanager BDI model.

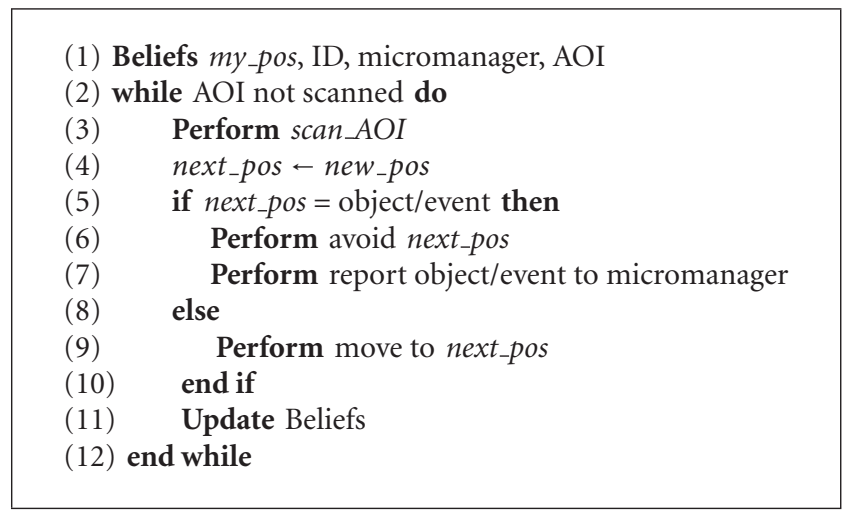

Algorithm 3: Sensing agent BDI model.

to systematic gaps in coverage, and missing target detection in the consequence.

(3) This algorithm is not adaptive to system/environment change and not robust to elements failure. Environmental change, adding or removing nodes will trigger system reconfiguration.

(ii) Fully Random Motion. In this mobility model, the sensing agent starts sweeping also from the top left corner, but it does not move in a fixed direction; instead, it picks a random position to move in it. Moreover it remembers its previously visited positions and prevents itself from these positions till certain aspiration criterion is met. In our case it waits about 5 seconds then it deletes the previously visited position. By this way the sensing agent can be forced to explore more in its environment and at the same time be prevented from getting stuck in a position where it is all surrounded with previously visited positions, thus increase the exploration and decrease the exploitation.

Figure 3 shows an example of the random motion of two sensing agents in a $10 \times 10$ environment. Again the green area is the agents specified AOI, and the blue dots are the recently visited positions which are removed after a certain time passes.

There are three main advantages of this strategy [40].

(1) This mobility model is easy to implement resulting in applications that deploy a large number of low

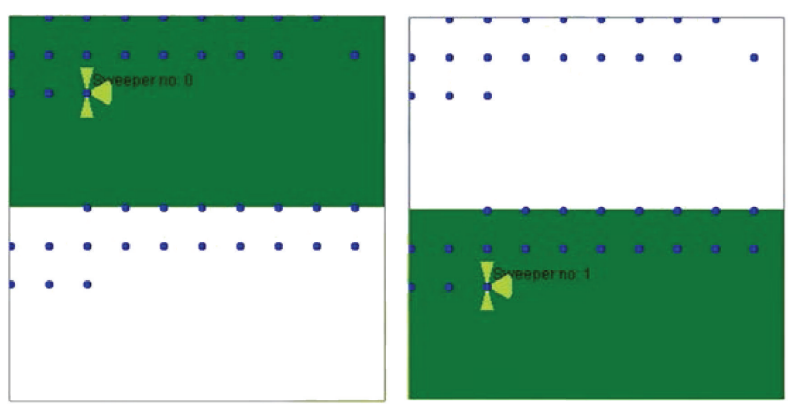

FIgUre 2: Two sensing agents in fully coordinated motion.

cost simple sensing agents. The system level missionspecific functions and performance requirements can be achieved through high nodes redundancy.

(2) This mobility model inherently adapts to dynamic environment where prior knowledge of the region of interest is not available.

(3) Because sensing agent path is not predictable, so the sensing agent cannot make use of its observations or knowledge to predict its path and thereby evade detection.

The disadvantage in this scanning mode is that there is no way to determine if the entire AOI has been scanned or not, so the stopping criteria must be time dependent, where the 


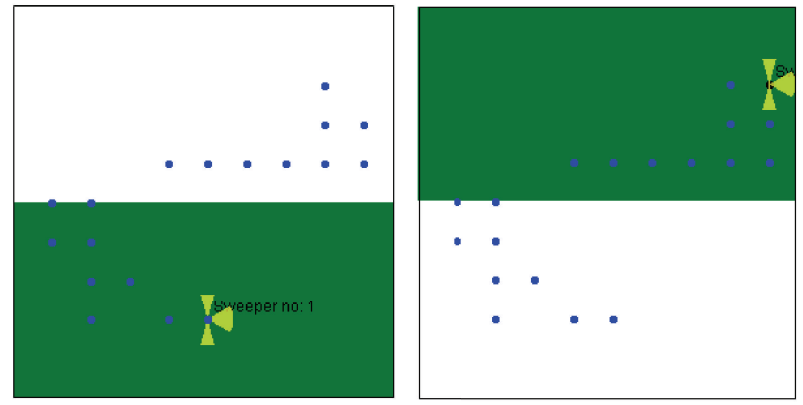

Figure 3: Two sweepers in fully random motion.

sensing agent is removed from the AOI after a certain time has passed.

4.4. Clustering. After the global map is formed by the macromanager, a number of acting agents are needed to manipulate the detected object/event. The number of detected objects/events is used to be higher than the number of acting agents. This raises the need to cluster the objects/events in the global map and assign each acting agent to a cluster of objects/events. Genetic algorithm (GA) is used to cluster the global map as shown in Algorithm 4. Dispersion (disp) is used as a similarity measure in this clustering technique. Dispersion is the distance between the acting agent and the objects/events assigned to it (the closer the objects/events is to the acting agent, the smaller the value of dispersion is and vice versa). The total dispersion is the summation of distances between all the acting agents and objects/events assigned to it.

In this population-based metaheuristic technique, there are a number of acting agents $\mathbf{i}$, location of objects $(\mathbf{x}, \mathbf{y})$, and number of objects $\mathbf{m}$. These input parameters can be represented as an array $\left\{(\mathbf{x}, \mathbf{y})_{1},(\mathbf{x}, \mathbf{y})_{2}, \ldots,(\mathbf{x}, \mathbf{y})_{\mathbf{m}}\right\}$, where $\mathbf{x}$ and $\mathbf{y}$ are the coordinates of the detected objects. The clustering algorithm outputs the set of detected objects/events in the global map associated with the corresponding acting agent as shown in Figure 4.

\section{Minefield Mapping as a Case Study}

The proposed approach is tested for validity with the real life problem of minefield mapping, as it requires cooperation between different robots to scan and clear a minefield from all the landmines.

5.1. Simulation Setup. Different development environments with different capabilities and levels of complexities are available for multirobot systems [1]. The proposed system has been implemented using Jadex, a BDI reasoning engine that allows for programming intelligent software agents in $\mathrm{XML}$ and Java.

(i) The Environment. In the proposed system, a simulation of a minefield called environment is created on a JADEX platform with four types of agents customized to our special case of demining system.
The size can be controlled and $10 \times 10$ up to $100 \times$ 100 environment sizes were tested.

(ii) The Sensing Agents. are autonomous mine sweeping robots equipped with a number of interoceptive (inner-state) and exteroceptive (outer-state) sensors. Interoceptive sensors include global positioning system (GPS) used to localize robots inside the mine field. Exteroceptive sensors are the mine detector used to detect nearby mines. There are three of them: one at front and one on each side of the robot. Also the robots are equipped with a wireless module to successfully communicate with the corresponding micromanager to send the individual mine map created from the sweepers. The sweeper has four beliefs about the environment and the system. It also has two goals to scan the AOI and report the object/events and finally two plans to execute these goals.

(iii) Sensor Model. The sensor is used by the sweeper to detect the mines in the environment. Two types of sensor models were used.

(1) Ideal Sensor: all the mine occurrences are detected by the sweeper. There is no probability of having overlooked nor unexpected detection.

(2) Noisy Sensor: to add realism to the simulation and make it as close as possible to the real life situation of sweeping. A random Gaussian noise is added to the ideal mine sensor and a certain value (sensitivity) is used to control if the sensor will detect the mine or not. If the random number is greater than this preset sensitivity value, the sensor will detect the mine. But if the random number is less than the sensitivity, the mine will not be detected and may cause the sweeper to explode.

(iv) The Micromanager. It is an agent that can be either running on the base station with no physical body or can be an unmanned aerial vehicle (UAV) which can monitor the environment and at the same time be safe from the dangers in the minefield. In both cases it has to be equipped also with wireless communication module to communicate with the other agents in order to receive the individual mine map from the sweepers and send the combined local mine map of its specific ROI to the macromanager. The micromanager has three beliefs about the system. It also has two goals: to divide the ROI into smaller AOI and report the object/events map, and finally it has two plans to execute these goals.

(v) The Macromanager. It is an agent running on the base station where it can communicate with the micromanagers and receive the local mine maps from them and compile the global mine map then cluster it according to the number of available acting agents or deminers. The macromanager has three beliefs about and the system. It also has three goals: to divide the 


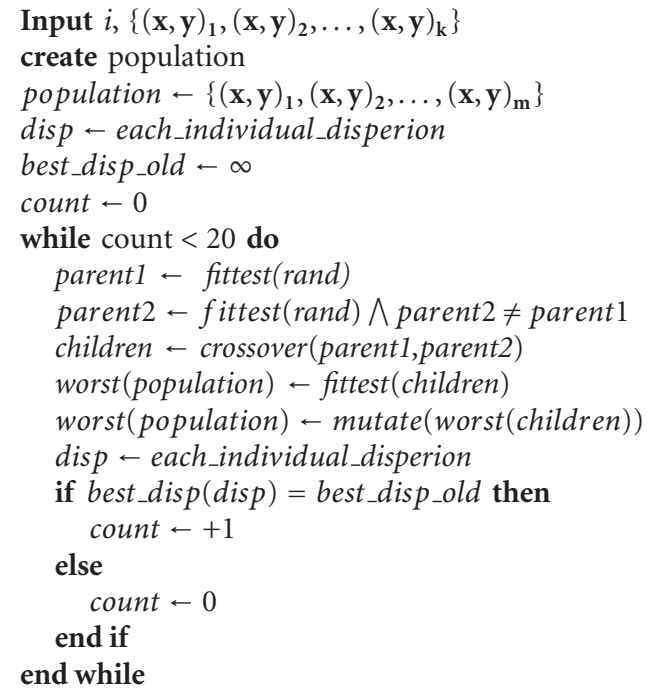

Algorithm 4: Genetic Algorithm (GA) for clustering.

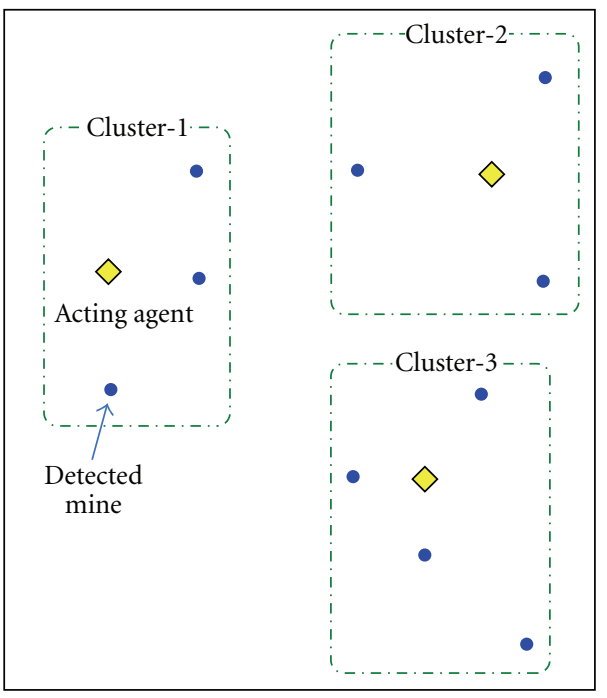

Figure 4: Clustered mine map.

environment into smaller ROI, to report the global object/events map to the user and to cluster the final map, and finally, it has three plans to execute these goals.

(vi) The Acting Agents. are the deminers that will be used to destroy or deactivate the detected mines. These acting agents can be, but are not limited to, automated/teleoperated flairs/rollers/plows or deactivation robots or any other robot capable of destroying/deactivating a certain target in a given map.

(vii) The Mines. In our simulation the number of mines is controlled and three cases for their distribution in the environment are proposed.
(1) Randomly Distributed Mines. the mines positions are fixed and are created randomly at the beginning of the simulation without any pattern. This represents the case where the user has no initial plan of how the mines are distributed in the minefield.

(2) Pattern Based Mines. the mines positions are also fixed and are created once but according to a predefined pattern. This is the most practical case because during previous demining processes after wars and conflicts certain patterns of distribution were noticed. For example, a typical Vietnamese protective minefield (as used in Cambodia between 1979-1989) might consist of two rows of antipersonnel mines, with $1 \mathrm{~m}$ spacing between each mine, and $1 \mathrm{~m}$ spacing between the mine rows [11].

(3) On-the-Fly Mines. the mines are being created during the ongoing simulation which represents the case when the mines are initially buried and undetectable but are now detectable due to the changing weather conditions. This model can be used simultaneously with any of the other two models. The rate of mines appearing on the surface can be controlled and it is called tickrate. However this model is more difficult than the other ones because number of mines and completion time will be correlated.

5.2. Evaluation Metrics. The measurement of effectiveness (MOE) of a scanning system can be gathered through the following two approaches. One approach is with objective related to deployment coverage [41-43]. Another approach is with the objective related to picture compilation $[1,44]$. 
TABle 1: Detection table.

\begin{tabular}{lcc}
\hline Actual state & Positive & Negative \\
\hline True & True-Positive (TP) & True-Negative (TN) \\
False & False-Positive (FP) & False-Negative (FN) \\
\hline
\end{tabular}

5.2.1. Normalized Cumulative Area Coverage. Cumulative area coverage is accumulation of the instantaneous area coverage between any two time instances, and cumulative area coverage of a mobile scanning system is the fraction of the geographical area covered by at least one sweeper at least one time within a certain time interval, denoted as $\mathrm{Ac}(t)$. The normalized area coverage is the percentage of cumulative area coverage to the total environment size to be scanned, thus the optimum values should be in the range of $100 \%$.

5.2.2. Measuring Picture Compilation. When sensing model of each sensor is given, injecting targets (objects or events) is a realistic method to evaluate the performance and working status of a whole scanning system. Results of target detection can not only assess system performance from an end-user point of view, but also address system characteristics and highlight algorithm weakness for further improvements. The following various metrics help to analyze the diverse aspects of scanning system $[44,45]$.

In the field of target detection and in the case of imperfect sensors, the performance is evaluated using these measures: precision, sensitivity, and $F$-measure $[45,46]$.

In order to explain the definition of these measurements, consider a cross contingency matrix in Table 1 showing a detection result of two categories (positive and negative category). TP is the number of targets that are the correctly detected. FP is the number of targets that are missed. TN is the number of false alarm. FN is the number of objects that do not belong to targets and are correctly not detected.

Formulas (4) and (5) give the definition of precision and Sensitivity according to the terms in Table 1. Precision is defined as the percentage of the number of correctly detected positive objects compared to the total number of detect $(\mathrm{TP}+\mathrm{TN})$, while sensitivity is defined as the percentage of the number of correctly detected positive objects compared to the number of all ground truth positive objects (TP + $\mathrm{FP})$. The $F_{\text {measure }}$ is a harmonic mean of the precision and sensitivity defined in Formula (6):

$$
\begin{gathered}
\text { Precision }=\frac{\mathrm{TP}}{\mathrm{TP}+\mathrm{TN}}, \\
\text { Sensitivity }=\frac{\mathrm{TP}}{\mathrm{TP}+\mathrm{FP}}, \\
F_{\text {measure }}=\frac{2 \times \text { Precision } \times \text { Sensitivity }}{\text { Precision }+ \text { Sensitivity }} .
\end{gathered}
$$

5.2.3. Completion Time. The time needed to complete the scanning process is also recorded to compare the different types of mobility models and time required for each to finish.

The time is measured between the start of sweeping of the first sweeper till the end of sweeping for the last sweeper, hence, the time is not only the time taken by one sweeper but rather the union of all the time taken by all sweepers $\mathbf{w}$ as shown in the followin formula:

$$
\text { Time }_{\text {all }}=\bigcup \text { Time }\left._{i}\right|_{i=1 \rightarrow \mathbf{w}} .
$$

5.3. Experiment Setup. Several experiments were conducted on the JADEX platform with different environment sizes and different number of sweepers and micromanagers. starting from 1 sweeper and 1 micromanager in a $10 \times 10$ area up to 50 sweepers and 10 micromanagers in $100 \times 100$ area.

The experiments focused on three pairs of models.

(1) Mobility models by testing coordinated and random motions.

(2) Mine distribution models by testing randomlydistributed and pattern-based mine distribution.

(3) Sensor model by changing the sensitivity of the mine detecting sensor.

Regarding the mobility models, the stopping criterion of the coordinated motion is to scan the entire environment and this execution time was recorded. However the stopping condition in random motion was the execution time required by this number of sweepers in this area size in the coordinated motion to finish the scanning. This way it can be guaranteed that both cases have the same chances to scan the environment. Moreover, this implies that completion time cannot be used as comparative measure because it has the same values in both cases. Both simulations were executed using randomly distributed mines model and noisy sensor model.

Regarding the mine distribution models, the coordinated motion is used to scan each environment twice, first for the randomly distributed mines model and second for the pattern-based mines model.

The pattern used in this simulation is similar to the one used by the German-Italian armies at the battle of Al Alamein as discovered by the Allied forces [47]. The pattern uses equally spaced mines and equally spaced rows of mines but with one cell shift between each row and the next. Figure 5 shows the pattern for $20 \times 20$ area; the spacing between the mines is 6 and the distance between each row and the other is 2 .

When this minefield is seen from an aerial view, it will be viewed as parallel diagonal-like lines. Finally one random mine is intentionally not deployed according to the pattern to simulate any possible error in the pattern during the mines deployment.

Regarding the sensor model, the sensor is modeled once as an ideal sensor that detects all the occurrences of the mines. The other case the sensor is modeled as a noisy sensor so that some occurrences may not be detected by the sweeper. The random mine distribution model is used in both models.

Each simulation was executed at least three times and the time needed to complete the sweeping, total area scanned and the precision, sensitivity and $F_{\text {measure }}$ were recorded in each simulation, then the average of all three simulations was studied. 


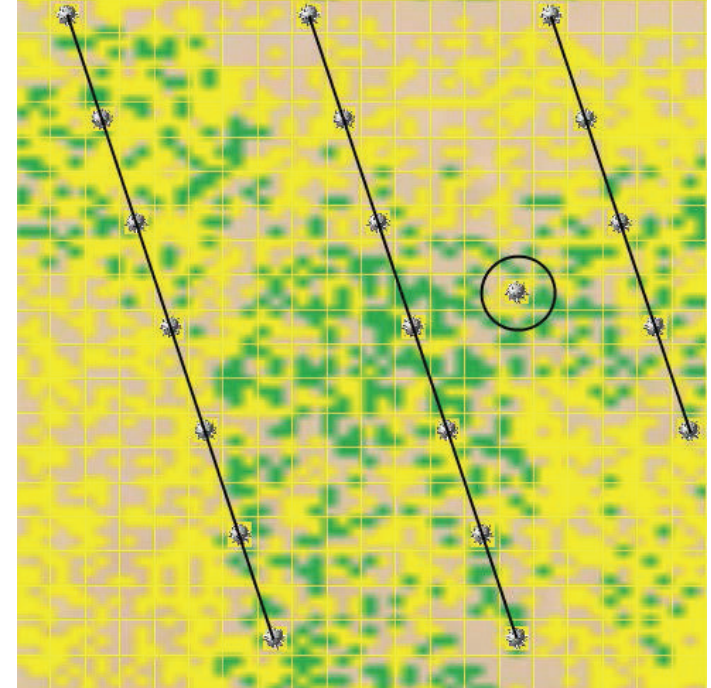

FIgURE 5: Pattern-based mine distribution in a $20 \times 20$ minefield.

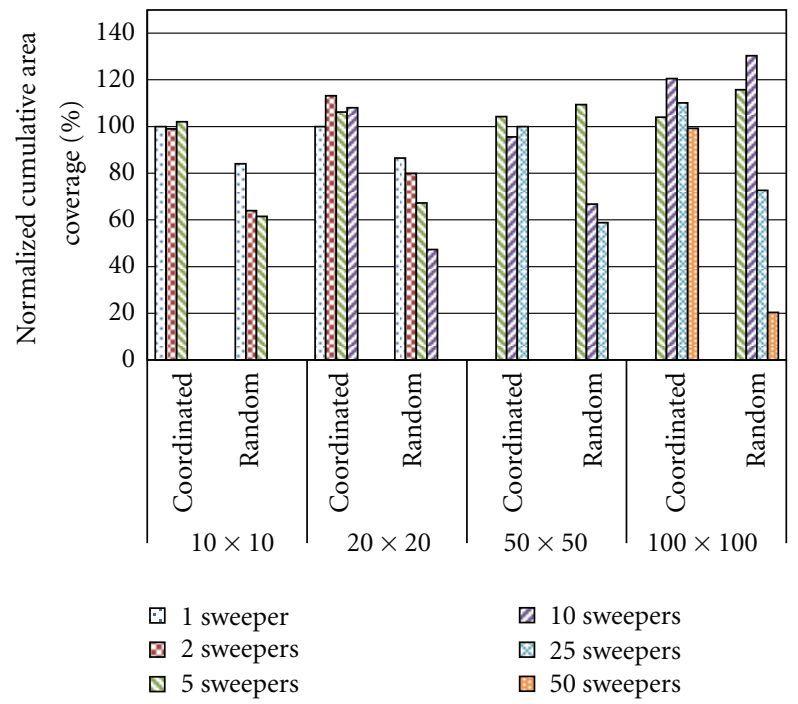

Figure 6: Area coverage in coordinated versus random motion model.

\section{Results and Discussion}

6.1. Mobility Models. The results from the coordinated motion and random motion are compared with each other to get better understanding of how the system works when increasing the area size and number of sweepers.

Normalized Cumulative Area Coverage. Figure 6 shows the normalized cumulative area coverage results of every number of sweepers for every area size depending on the type of motion coordinated and random. The normalized cumulative area coverage measured is not absolute, meaning that if the sweeper scans the same position twice, it is counted as two separate areas. (i) Fully Coordinated Motion. The normalized cumulative area coverage increases as the number of sweepers increases. However it must be noted that if the cumulative area covered is larger than the total area to be scanned, this implies that one of the sweepers was exploded and the other sweepers had to scan its AOI one more time thus also increasing the completion time.

(ii) Random Motion. The figure shows that the random model fails to scan the environment effectively and even decreases with the increase of the area size. This occurs because when the area size increases, the number of mines increases thus the probability of sweeper failure due to undetected mine increases. Also, the results show that the area coverage increases as the number of used sweepers increases, but this is valid only for small area sizes. The interpretation to that is that for large areas and many sweepers the $Y$-axis is divided upon the number of sweepers hence ratio between the length and width of the assigned AOI become very small. This decreases the probability of allowing the sweeper to explore new positions in the $X$-axis and if it is required to overcome this problem by increasing the probability of scanning in the $X$-axis the motion will not be truly fully random. A better solution to this problem is to divide the environment into squares or rectangles with reasonable length-to-width ratio, however, it may face problems during practical implementation.

F-Measure. Figure 7 shows the results depending on the type of motion coordinated and random.

(i) Fully Coordinated Motion. In Figure 8, the F-measure represents the amount of error in the sensitivity of the sensors of all the sweepers, hence if the number of sweepers is large enough to compensate the loss of one or more sweepers, the F-measure will increase. But if the number of sweepers is a small number, then the loss of sweepers will greatly affect the system performance as shown in the case of $50 \times 50$ area.

(ii) Random Motion. On the other hand, the F-measure increases with the increase of environment size and number of sweepers for small area sizes, but decreases greatly for large area sizes because the $F$-measure faces the same problem of exploring in the $\mathrm{X}$-direction similar to the area coverage. Thus the $F$-measure decreases as the environment size and the number of sweepers increase as shown in the case of $50 \times$ 50 and $100 \times 100$ area.

Completion Time. No need to compare the completion time needed because the same values obtained in the coordinated motion were used in the random motion.

As expected the results show that the completion time is inversely proportional to the number of sweepers used in scanning. However this relation is not linear because in the case of small number of sweepers the AOI assigned to each one is relatively large and if any sweeper explodes, the AOI assigned to each sweeper becomes even larger and sweeping starts from the beginning again. Thus it is recommended to 


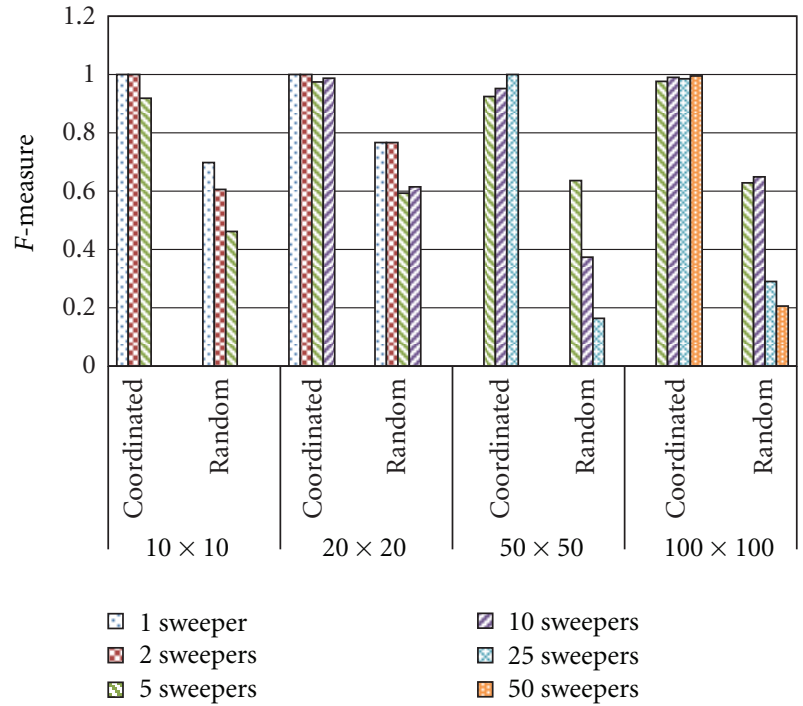

FIGURE 7: F-measure in coordinated versus random motion model.

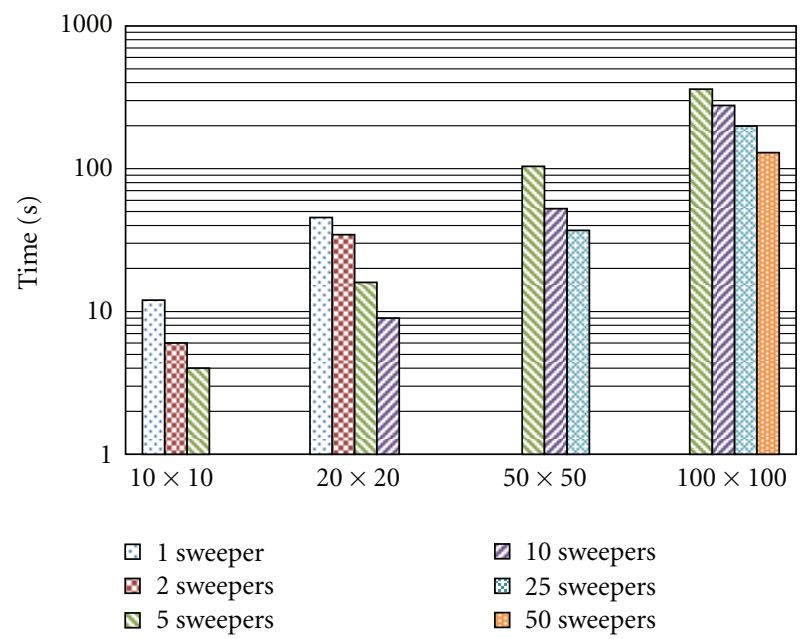

Figure 8: Time in fully coordinated motion.

balance the number of sweepers relative to the environment size.

6.2. Mine Distribution Models. The results from both types of mine distribution models are compared with each other to study the effects increasing the area size and number of sweepers on the system.

Normalized Cumulative Area Coverage. Figure 9 shows the results of every number of sweepers for every area size depending on the type of mines distribution.

Normalized cumulative area coverage is observed to be increasing more in the pattern-based distribution as the number of sweepers increases, but for large areas the area coverage increases beyond the total area to be scanned thus duplication of effort has occurred due to sweeper failure. Because now many mines are located on the same row, so

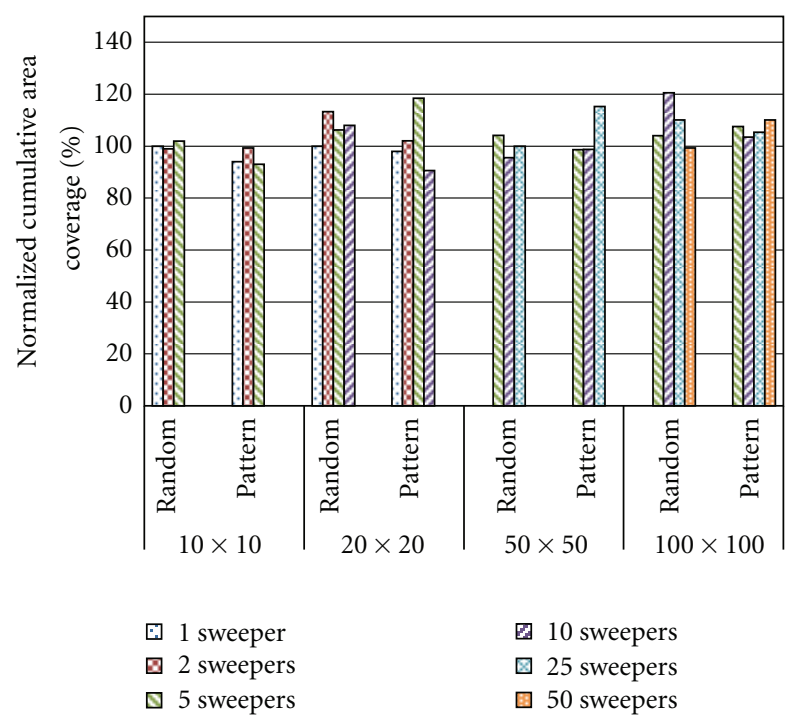

FIGURE 9: Area coverage in random versus pattern-based distribution model.

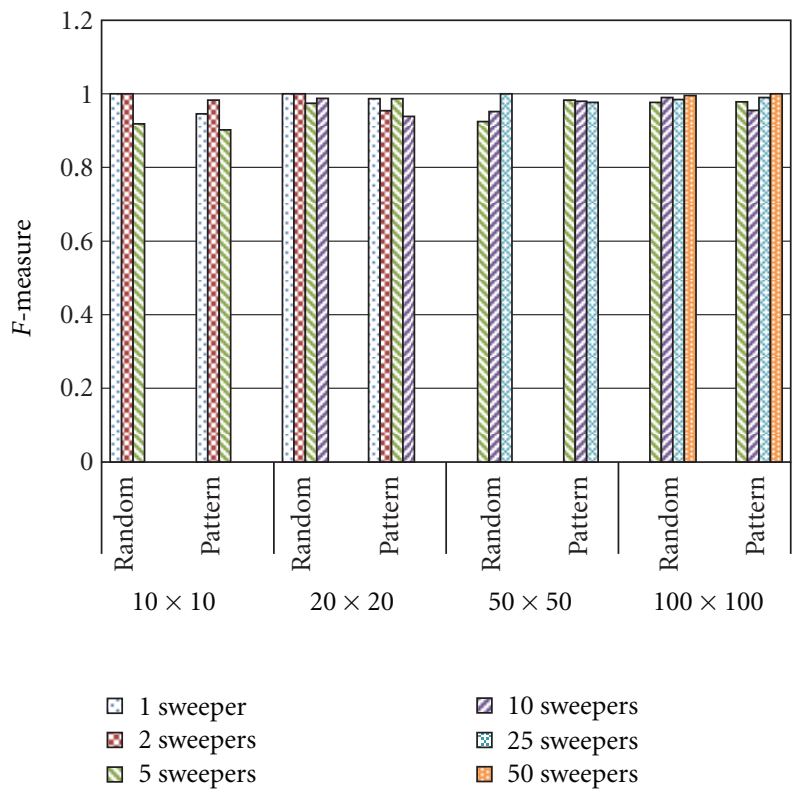

FIGURE 10: $F$-measure in random versus pattern-based distribution model.

there is a high chances of failure in a certain row but low chances in other rows. When failure occurs, environment has to be rescanned again by the remaining sweepers.

F-Measure. Figure 10 shows the results depending on the type of mines distribution. As explained in the area coverage, the $F$-measure will increase with the increase of number of sweepers because the environment is rescanned and ensured that no mines were skipped due to the failures.

Completion Time. Figure 11 shows the results depending on the type of mines distribution. The graphs show that 


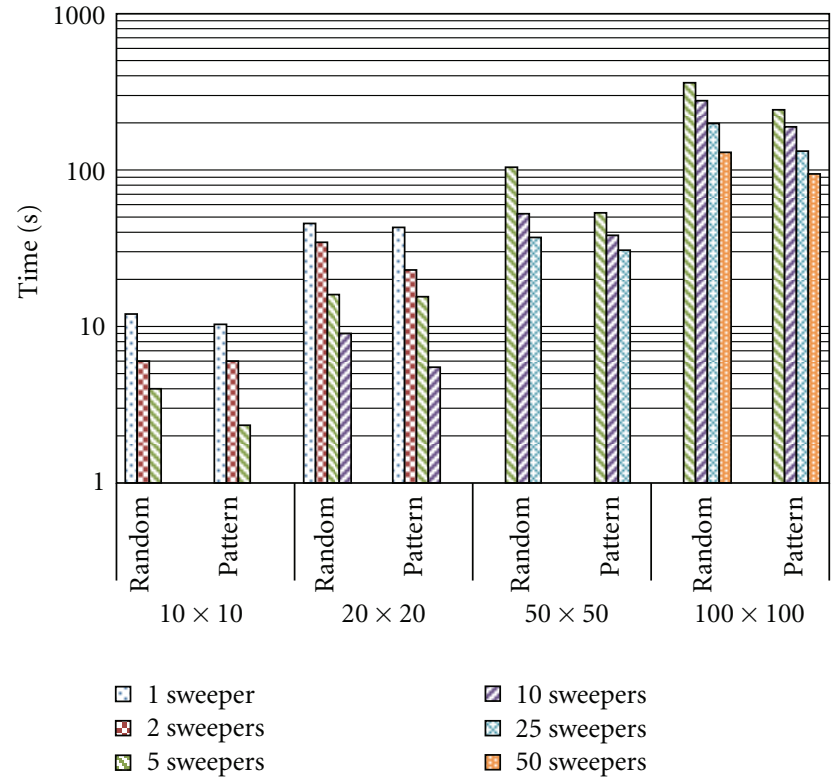

FIgURE 11: Time in random versus pattern-based distribution model.

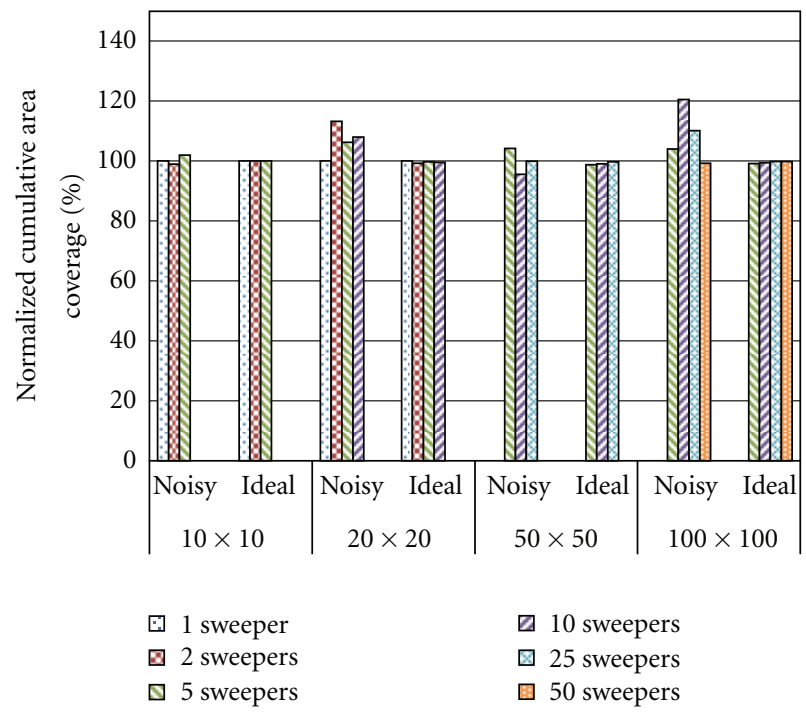

FIGURE 12: Area coverage in noisy versus ideal sensor model.

in many cases the time needed for the pattern-based distribution is less than that of the randomly distributed. The reason for this is because in the pattern-based minefield the mines are equally spaced and shifted in rows so there are very small probability for two sweepers reporting to the same micromanager at the same time. This gives the micromanager more time to process each report separately instead of processing many reports at the same time thus saving time in the entire process. In all the cases it was noticed that when the number of sweepers assigned to each micromanager is large, the efficiency of the system in terms of completion time and $F$-measure decreases greatly.
6.3. Sensor Models. The results from both types of sensor models are compared with each other to study the effects increasing the area size and number of sweepers on the system.

Normalized Cumulative Area Coverage. Figure 12 shows the results of every number of sweepers for every area size depending on the type of sensor used.

Normalized cumulative area coverage of the accurate sensor is observed to be less than that of the noisy sensor. This is expected because there is no probability of sweeper failure and duplication of the scanning. So the value remains almost constant in all experiments.

F-Measure. Figure 13 shows the results depending on the type of sensor used. The $F$-measure of the ideal sensor is always greater than the noisy sensor and almost a constant 1 showing that there are no undetected mines or unexpected results. except for high sweeper to micromanager ratio (50 sweepers in $100 \times 100$ ) because the micromanager was not able to process all the reports simultaneously.

Completion Time. Figure 14 shows the results depending on the type of sensor used. The results show that the time for the ideal sensor is always less than that of the noisy sensor again because no sweeper failure will occur and each sweeper is assigned to a smaller AOI. Therefore the ideal sensor gives the optimum results for the area coverage, $F$-measure, and time.

6.4. Discussion. The challenging problems in the implementation were the coordination between the different kinds of agents because each agent has its own set of beliefs, desires, and intentions and the actions of some agents are dependent on the beliefs of other agents of different types.

Another challenging problem was the implementation of the different mobility models required to handle all the possible cases of the mine location relative to the sweeper and decide how the sweeper will be able to avoid the mine and continue scanning of the environment. Another problem was how to reassign the environment on the remaining sweepers one more time to handle the case of sweeper failure.

The interpretation of the results indicates that using the coordinated motion with moderate number of sweepers relative to the environment size is the most efficient method of scanning; however, if the disadvantages of the coordinated motion (described earlier) cannot be avoided, then the random motion can be used but with limited number of sweepers and considering the effects of the length to width ratio of each robot's AOI.

It was observed that scanning for pattern-based mines will take less time although it may include duplication of scanning in some cases and will increase the $F$-measure. Also using accurate and precise sensors to detect the mines will save the robots from explosion thus prevent duplication of scanning and decrease the completion time and give the optimum results. The results of all models for the worst case 
TABLE 2: Mobility models.

\begin{tabular}{lccc}
\hline $100 \times 100$ area & \multicolumn{2}{c}{ Mobility model (coordinated/random) } \\
No. of sweepers & Area \% & F-measure & Time $($ sec) \\
\hline 5 & $104 / \mathbf{1 1 5 . 7}$ & $0.97 / \mathbf{0 . 6 2}$ & 361 \\
10 & $120.5 / 130.3$ & $0.989 / 0.64$ & 277.5 \\
25 & $110.1 / 72.6$ & $0.984 / 0.29$ & 198.5 \\
50 & $\mathbf{9 9 . 3 / 2 0 . 3}$ & $\mathbf{0 . 9 9 4 / 0 . 2}$ & 129.5 \\
\hline
\end{tabular}

Table 3: Mine distribution model.

\begin{tabular}{lccc}
\hline $100 \times 100$ area & \multicolumn{2}{c}{ Mine distribution (random/pattern) } & Time $($ sec) \\
No. of sweepers & Area \% & F-measure & $361 / 242.7$ \\
\hline 5 & $104 / 107$ & $0.97 / 0.97$ & $277.5 / 189.25$ \\
10 & $120.5 / \mathbf{1 0 3 . 5}$ & $0.989 / 0.95$ & $198.5 / 132.3$ \\
25 & $110.1 / 105.4$ & $0.984 / 0.989$ & $\mathbf{1 2 9 . 5 / 9 4 . 3}$ \\
50 & $\mathbf{9 9 . 3 / 1 1 0}$ & $\mathbf{0 . 9 9 4 / 0 . 9 9 4}$ & $\mathbf{1 2 9 4}$ \\
\hline
\end{tabular}

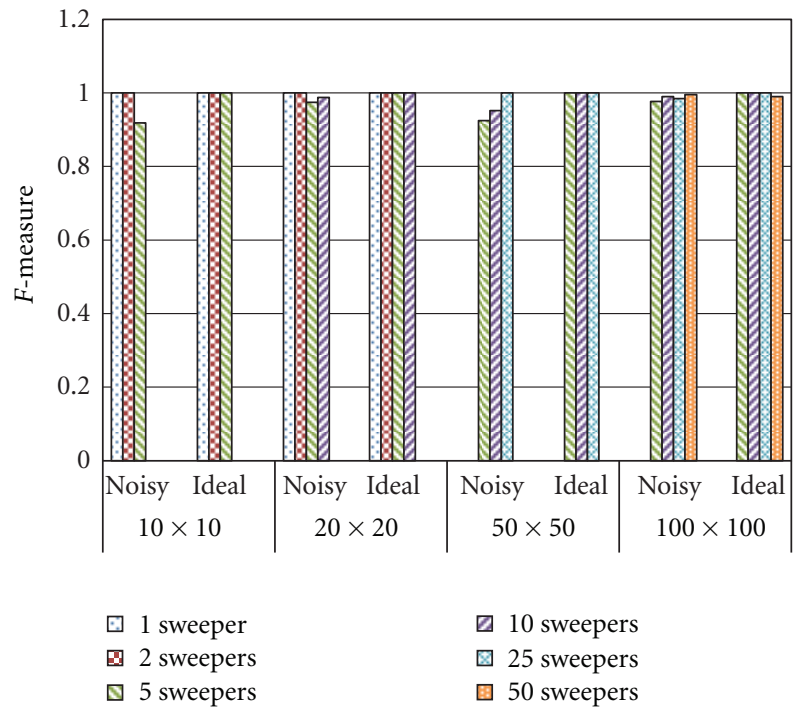

Figure 13: F-measure in noisy versus ideal sensor model.

which is the $100 \times 100$ area are shown in Tables 2, 3, and 4 . The best situation in each case is highlighted with bold font.

\section{Conclusion}

A cooperative multirobot system is proposed with builtin know-how and know-how-to-cooperate using the BDI model to control the actions of the robots and TeamLog theory to control the interactions between the various robots and the flow of information in the system. These models were selected because of their flexibility and their similarity with the human decision-making process. The proposed approach was tested with a practical case study of a humanitarian demining as it is considered a severe problem in many developing countries. The results showed the system's flexibility to errors in the environment like sensor's inaccuracy. the results also showed the scalability of

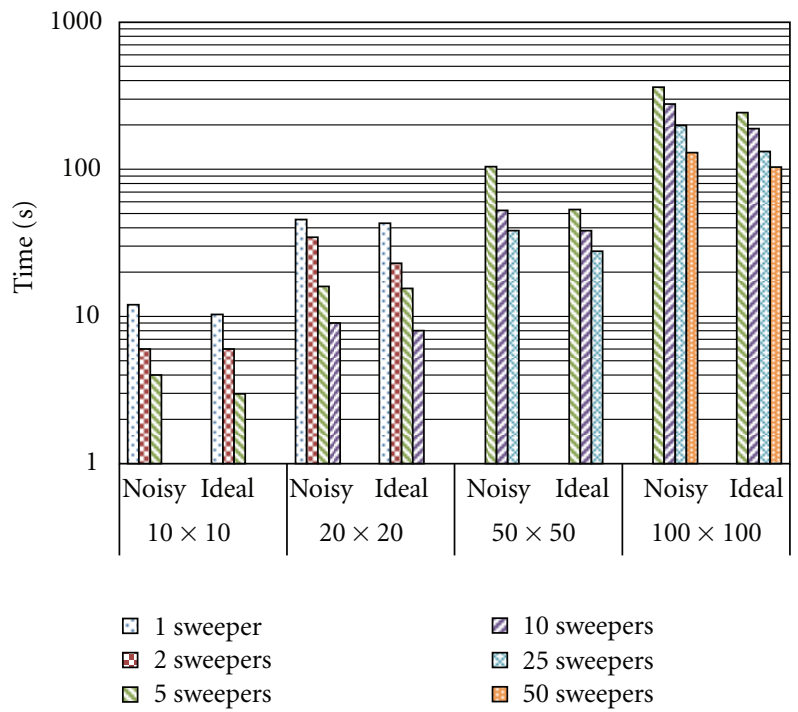

FIGURE 14: Time in noisy versus ideal sensor model. the system in controlling many agents up to 60 agents in 100 $\times 100$ environment in a robust manner.

The future aspects of research include studying other types of mobility models especially emergent mobility model where there is no formal coordination between the sweepers in the motion. Each sweeper observes how the other neighboring sweepers move and moves based on the collective motion to reach a certain destination. This approach will minimize the amount of communication between the sweepers and the micromanagers thus reducing the computing power needed in both agents.

Moreover, the proposed approach is expected to be applied on real robots in the national robotic competition "Minesweepers: Towards a Landmine-free Egypt" [10] that is organized by IEEE Robotics and Automation Society (RAS) Egypt Chapter and sponsored by the German University in Cairo in collaboration with the Executive Secretariat for 
TABLE 4: Sensor model.

\begin{tabular}{|c|c|c|c|}
\hline \multirow{2}{*}{$\begin{array}{l}100 \times 100 \text { area } \\
\text { No. of sweepers }\end{array}$} & \multicolumn{3}{|c|}{ Sensor model (noisy/ideal) } \\
\hline & Area \% & $F$-measure & Time (sec) \\
\hline 5 & $104 / 99.1$ & $0.97 / 1$ & $361 / 259$ \\
\hline 10 & $120.5 / 99.4$ & $0.989 / 1$ & $277.5 / 168$ \\
\hline 25 & $110.1 / 99.8$ & $0.984 / 1$ & $198.5 / 116$ \\
\hline 50 & $99.3 / 99.79$ & $0.994 / 0.989$ & $129.5 / 103.5$ \\
\hline
\end{tabular}

the Demining and Development of the North West Coast, Egyptian Ministry of International Cooperation.

\section{References}

[1] H. Irandoust, A. Benaskeur, and A. Khamis, "Cooperation in distributed surveillance: concepts, theories, models and technology enablers," Tech. Rep., Valcartier, 2010.

[2] L. Parker, "Multiple mobile robot systems," in Handbook of Roboitcs, pp. 921-941, Springer, 2008.

[3] D. Floreano and C. Mattiussi, Bio-Inspired Artificial Intelligence: Theories, Methods, and Technologies, MIT Press, 2008.

[4] A. Khamis, "Cooperative sensor and actor networks in distributed surveillance context," in Proceedings of the 10th International Conference on Practical Applications of Agent and Multiagent Systems (PAAM '12), Spain, 2012.

[5] U. Witkowski, M. El-Habbal, S. Herbrechtsmeier et al., "Ad-hoc network communication infrastructure for multirobot systems in disaster scenarios," in Proceedings of the EURON/IARP International Workshop on Robotics for Risky Interventions and Surveillance of the Environment, Benicassim, Spain, January 2008.

[6] J. Conesa-Munoz, A. Ribeiro, and G. Pajares, "A multi-path planning approach based on a genetic algorithm for a robot fleet working in arable crops," in Proceedings of the Portuguese Conference on Artificial Intelligence (EPIA '11), 2011.

[7] S. Verret, "Current state of the art in multirobot system," Tech. Rep. DRDC Suffield TM 2005-241, Technical Memorandum, 2005.

[8] P. Doherty and F. Heintz, "A delegation-based cooperative robotic framework," in Cooperative Information Agents, 2007.

[9] R. Cassinis, G. Bianco, A. Cavagi, and W. Ransenigo, "Landmines detection methods using swarms of simple robots," in Proceedings of the 6th International Conference on Intelligent Autonomous Systems, pp. 212-218, 2000.

[10] A. Khamis, "Minesweepers: towards a landmine-free Egypt," The Journal of ERW and Mine Action, no. 17.1, 2013.

[11] R. Keeley, "Understanding landmines and mine action," June 3, 2012, http://mit.edu/demining/assignments/understanding-landmines.pdf.

[12] A. Khamis, “16374- cooperative multi-robot systems,” Master course, Carlos III University of Madrid, 2011-2012.

[13] L. Giraldeau and T. Caraco, Social Foraging Theory, Monographs in Behavior and Ecology, Princeton University Press, 2000.

[14] H. Najjaran and A. A. Goldenberg, "Landmine detection using an autonomous terrain-scanning robot," Industrial Robot, vol. 32, no. 3, pp. 240-247, 2005.

[15] J. Vidal, Fundamentals of Multiagent Systems with NetLogo Examples, 2010.
[16] A. Benaskeur, A. Khamis, and H. Irandoust, "Augmentative cooperation in distributed surveillance systems for dense regions," International Journal of Intelligent Defence Support Systems, vol. 4, no. 1, pp. 20-49, 2011.

[17] A. Bond and L. Gasser, Readings in Distributed Artificial Intelligence, Morgan Kaufmann, 1988.

[18] N. R. Jennings, K. Sycara, and M. Wooldridge, "A roadmap of agent research and development," Autonomous Agents and Multi-Agent Systems, vol. 1, no. 1, pp. 7-38, 1998.

[19] G. Weiss, Multiagent Systems: A Modern Approach to Distributed Artificial Intelligence, MIT Press, 1999.

[20] M. Singh, A Theoretical Framework for Intetions, Know-How and Communication, Springer, 1994.

[21] M. P. Pacaux-Lemoine and S. Debernard, "Common work space for human-machine cooperation in air traffic control," Control Engineering Practice, vol. 10, no. 5, pp. 571-576, 2002.

[22] M. Bratman, Intention, Plans, and Practical Reason, CSLI Publications, 1999.

[23] M. Georgeff, P. B. Pell, M. Pollack, M. Tambe, and M. Wooldridge, "The belief-desire-intention model of agency," in Proceedings of the International Workshop on Intelligent Agents, 1998.

[24] M. Bratman, "What is intention?" in Intentions in Communication, p. 1532, MIT Press, Cambridge, Mass, USA, 1990.

[25] S. Bruecjner and H. V. D. Parunak, "Extrapolation of the opponent's Pas behaviours," in Adversarial Reasoning: Computational Approaches to Reading the Opponents Mined, 2008.

[26] N. Jennings, "Towards a cooperation knowlege level for collaborative problem solving," in Proceedings of the 10th European Conference on Artificial Intelligence, pp. 224-228, Vienna, Austria, 1992.

[27] F. Brazier, C. Jonker, and J. Treur, Formalization of a Cooperation Model Based on Joint Intentions, Lecture Notes in AI, Springer, 1997.

[28] V. Hilaire, O. Simonin, A. Koukam, and J. Ferber, "A formal approach to design and reuse agent and multiagent models," in Proceedings of the 5th International Workshop on AgentOriented Software Engineering V (AOSE '04), pp. 142-157, July 2004.

[29] J. Lehman, J. Laird, and P. Rosenbloom, "A gentle introduction to soar: an architecture for human cognition," in Invitation to Cognitive Science, MIT Press, 1996.

[30] B. Dunin-Keplicz and R. Verbrugge, Teamwork in Multi-Agent Systems: A Formal Approach, Wiley, 2010.

[31] R. Fagin, J. Halpern, Y. Moses, and M. Vardi, Reasoning about Knowledge, MIT Press, 1995.

[32] J.-C. Meyer and W. van der Hoek, Epistemic Logic for AI and Theoretical Computer Science, Cambridge University Press, Cambridge, UK, 1995.

[33] P. R. Cohen and H. J. Levesque, "Intention is choice with commitment," Artificial Intelligence, vol. 42, no. 2-3, pp. 213261, 1990. 
[34] B. Dunin-Kęplicz and R. Verbrugge, "Collective intentions," Fundamenta Informaticae, vol. 51, no. 3, pp. 271-295, 2002.

[35] E. Graedel, "Why is modal logic so robustly decidable?" Bulletin of the European Association for Theoretical Computer Science, vol. 68, pp. 90-103, 1999.

[36] B. Horling and V. Lesser, "A survey of multi-agent organizational paradigms," Knowledge Engineering Review, vol. 19, no. 4, pp. 281-316, 2004.

[37] D. Gage, "Command control for many-robot systems," in Proceedings of 19th Annual AUVS Technical Symposium, pp. 22-24, 1992.

[38] D. Gage, "Randomized search strategies with imperfect sensors," in Mobile Robots VIII, vol. 2058 of Proceedings of SPIE, pp. 270-279, 1993.

[39] Y. Q. Miao, A. Khamis, and M. Kamel, "Coordinated motion control of mobile sensors in surveillance systems," in Proceedings of the 3rd International Conference on Signals, Circuits and Systems (SCS '09), November 2009.

[40] M. Yun-Qian, A study of mobility models in mobile surveillance systems [M.S. thesis], University of Waterloo, 2010.

[41] M. Cardei and J. Wu, Coverage in Wireless Sensor Networks, CRC Press, 2004.

[42] S. Meguerdichian, F. Koushanfar, M. Potkonjak, and M. B. Srivastava, "Coverage problems in wireless ad-hoc sensor networks," in Proceedings of the 20th Annual Joint Conference of the IEEE Computer and Communications Societies, pp. 13801387, April 2001.

[43] B. Liu, P. Brass, O. Dousse, P. Nain, and D. Towsley, "Mobility improves coverage of sensor networks," in Proceedings of the 6th ACM International Symposium on Mobile Ad Hoc Networking and Computing (MOBIHOC '05), pp. 300-308, May 2005.

[44] B. Liu and D. Towsley, "On the coverage and detectability of large-scale wireless sensor networks," in Proceedings of the Modeling and Optimization in Mobile, Ad Hoc and Wireless Networks Conference, 2003.

[45] A. T. Nghiem, F. Bremond, M. Thonnat, and V. Valentin, "ETISEO, performance evaluation for video surveillance systems," in Proceedings of the IEEE Conference on Advanced Video and Signal Based Surveillance (AVSS '07), pp. 476-481, September 2007.

[46] R. Duda, P. Hart, and D. Stork, Pattern Classification, WileyInterscience, 2000.

[47] W. D. O. of the Chief of Engineers, German minefields at alamein 1943, http://cgsc.cdmhost.com/cdm/singleitemlection/p4013coll8/id/1336/rec/14. 

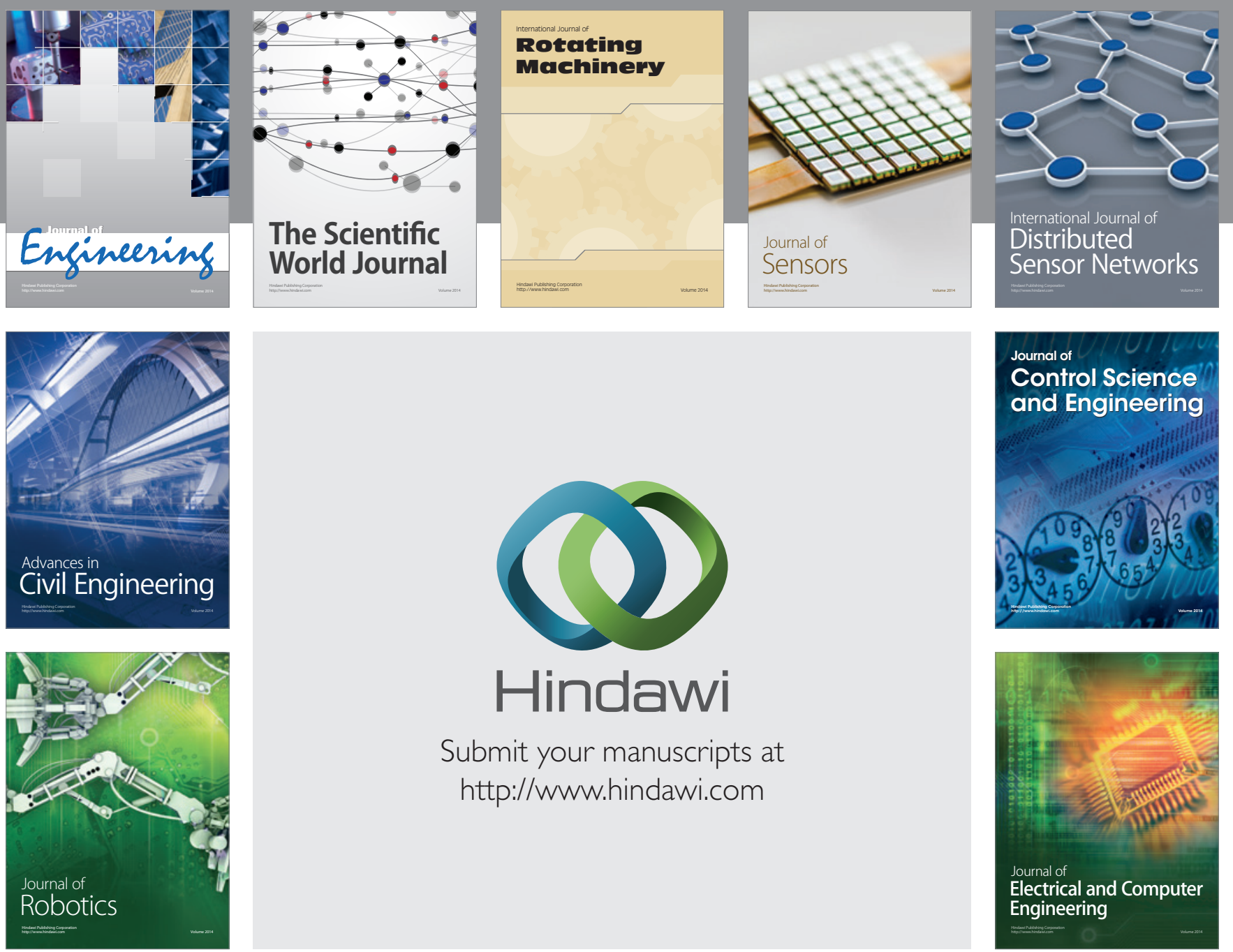

Submit your manuscripts at

http://www.hindawi.com
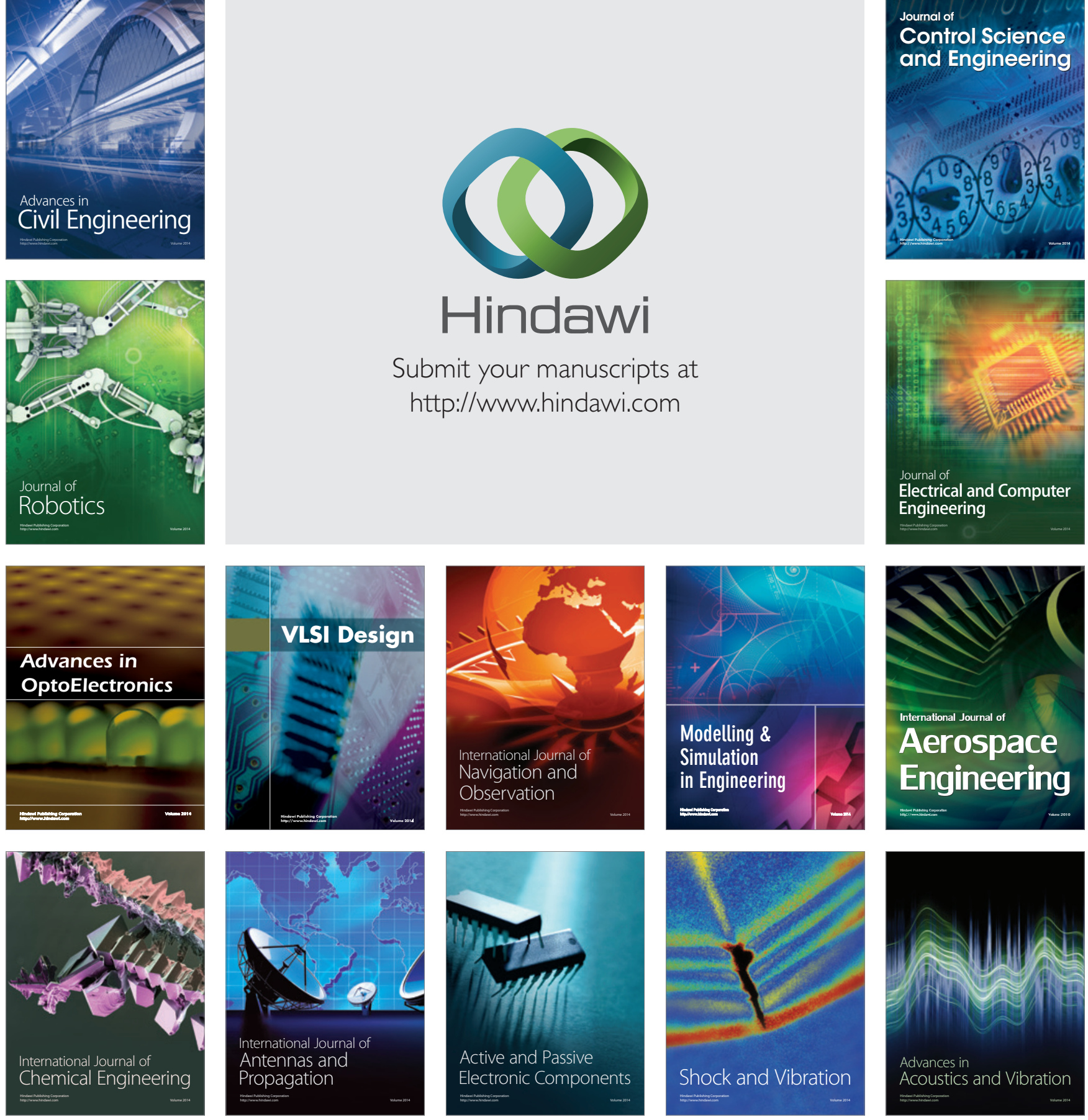